propylene mesh graft was introduced through the 10$\mathrm{mm}$ trocar and tacked with spiral tackers. The patient could be discharged the next day after requiring only minimal analgesics. At this writing, $2 \frac{1}{2}$ years after the operation, there is no sign of recurrence.

This Grynfeltt hernia could safely be treated using the extraperitoneal approach, which obviates opening and closing the peritoneum, thereby reducing operative time and possibly postoperative complications.

Key words: Lumbar herina - Grynfeltt hernia Retropitoneal endoscopic repair - Prosthetic mesh graft

Correspondence to: R. R. Postema

\section{Hand-assisted laparoscopic microwave coagulation therapy for hepatocellular carcinoma}

\author{
N. Yamashiki, ${ }^{\prime}$ T. Kato, ${ }^{2}$ R. W. Bailey, ${ }^{3}$ A. Tekin, ${ }^{2}$ \\ J. I. Moon, ${ }^{2}$ D. Levi, ${ }^{2}$ K. R. Reddy, ${ }^{1}$ E. R. Schiff, ${ }^{1}$ \\ A. G. Tzakis ${ }^{2}$ \\ ${ }^{1}$ Division of Hepatology, Department of Medicine, University of \\ Miami, School of Medicine, $1500 \mathrm{NW}$ 12th Avenue, Suite 1101, \\ Miami, FL 33136, USA \\ ${ }^{2}$ Division of Transplantation, University of Miami, School of \\ Medicine, 1801 NW 9th Avenue, Suite 508, Miami, FL 33136, USA \\ 3 Division of Laparoscopic Surgery, Department of Surgery, \\ University of Miami, School of Medicine, 1801 NW 9th Avenue, Suite \\ 508, Miami, FL 33136, USA \\ Received: 2 July 2001/Accepted in final form: 5 September 2001/Online \\ publication: 14 January 2002 \\ DOI: $10.1007 / \mathrm{s} 00464-001-4136-7$
}

\section{Abstract}

Microwave coagulation therapy has been used for local ablation of hepatocellular carcinoma in the setting of liver cirrhosis. This technique can be performed laparoscopically when the tumor is located on the surface of the liver or attached to other intraabdominal organs. However, a laparoscopic approach becomes difficult when it is necessary to mobilize the liver or lyse adhesions to gain access to the tumor. We used a hand-assisted laparoscopic technique for the application of microwave coagulation therapy in two cirrhotic patients with hepatocellular carcinoma to ensure safe mobilization of the liver and adhesiolysis. The first patient presented with a $4.5-\mathrm{cm}$ mass in the left lobe of the liver that was densely adherent to the lesser omentum. The use of a hand-assist device allowed us to perform a safe exploration, adhesiolysis, and subsequent tumor ablation. In the second patient, the tumor was located on the posterior aspect of the right lobe of the liver. The handassist device allowed safe mobilization of the liver and exposure of the tumor. No significant postoperative complications were noted in either patient. Complete ablation of the tumors was confirmed with contrastenhanced computed tomography scanning. In conclusion, hand-assisted laparoscopy is an effective adjunct to microwave ablation therapy in patients with hepatocellular carcinoma and liver cirrhosis. It enables the safe completion of the procedure under laparoscopic guidance, thereby averting laparotomy.
Key words: Hepatocellular carcinoma - Microwave ablation therapy - Hand-assisted laparoscopic surgery Correspondence to: $\mathrm{T}$. Kato

\section{Spillage of stones from the gallbladder during laparoscopic cholecystectomy and complication of a retroperitoneal abscess mimicking gluteal abscess in elderly patients}

\section{Dashkovsky, J. C. Cozacov}

Department of Surgery, Sieff Government Hospital, Safed, Israel Received: 17 September 2001/Accepted in final form: 4 October 2001/ Online publication: 11 January 2002

DOI: $10.1007 / \mathrm{s} 00464-001-4233-7$

\section{Abstract}

Gallbladder perforation during laparoscopic cholecystectomy with spillage of bile and gallstones occurs in a substantial percentage of patients (up to $40 \%$ ). We report the case of a 77-year-old woman who presented with fever of unknown etiology and a complication of retroperitoneal abscess mimicking a gluteal abscess with gallstones and clips the abscess. Spillage of gallstones from perforation of the gallbladder is a well-recognized complication of laparascopic cholecystectomy, especially several months after the initial surgery, as in the reported case.

Key words: Laparoscopic cholecystectomy - Gallstones spillage - Gluteal abscess

Correspondence to: I. Dashkovsky-Email: drigor@ matav.netnet.il

\section{Splenic cysts}

\section{A new approach to partial splenectomy}

\section{C.-M. Ho}

Department of Surgery, Hsin-Chu Hospital, Department of Health, The Executive Yuan, No. 25, 442nd Lane, Section 1, Chin-Kou Road, Hsin-Chu, Taiwan

Received: 9 October 2001/Accepted in final form: 23 October 2001/

Online publication: 11 January 2002

DOI: $10,1007 / \mathrm{s} 00464-00 !-4111-3$

\section{Abstract}

Laparoscopic partial splenectomy of a splenic cyst in a 19 -year-old female patient is discussed. We successfully performed a laparoscopic partial splenectomy and achieved meticulous hemostasis using an Endo-GIA stapling device. The patient tolerated the procedure well and was discharged on postoperative day 2. At this writing, she has been followed for 30 months without cyst recurrence. Minimally invasive surgery provides an alternative to conventional surgical procedures, with a short hospital stay and without the added morbidity of laparotomy.

Key words: Laparoscopic - Partial splenectomy Splenic cyst

Correspondence to: C. M. Ho 